\title{
Prim's Algorithm for Optimizing Fiber Optic Trajectory Planning
}

\author{
Muhammad Iqbal $^{1}$, Andysah Putera Utama Siahaan ${ }^{2}$, Nathania Elizabeth Purba ${ }^{3}$, Dedi Purwanto ${ }^{4}$ \\ ${ }^{1,2,4}$ Faculty of Computer Science, Universitas Pembangunan Panca Budi, Medan, Indonesia \\ ${ }^{3}$ Faculty of Computer Science and Information Technology, Universitas Sumatera Utara, Medan, Indonesia \\ ${ }^{2}$ Ph.D. Student of School of Computer and Communication Engineering, Universiti Malaysia Perlis, Kangar, Malaysia
}

\begin{abstract}
The transition of copper cable technology to fiber optic is very triggering the development of technology where data can be transmitted quickly and accurately. This cable change can be seen everywhere. This cable is an expensive cable. If it is not installed optimally, it will cost enormously. This excess cost can be used to other things to support performance rather than for excess cable that should be minimized. Determining how much cable use at the time of installation is difficult if done manually. Prim's algorithm can optimize by calculating the minimum spanning tree on branches used for fiber optic cable installation. This algorithm can be used to shorten the time to a destination by making all the points interconnected according to the points listed. Use of this method helps save the cost of fiber optic construction.
\end{abstract}

Keywords : Fiber Optic, Prim's Algorithm, Shortest Path

\section{INTRODUCTION}

Fiber Optic is a thing that has become the current technology needs, especially now where the needs of communication and technology are getting higher. It is used primarily for the internet in all places, at home, at the office or in public areas. Substitution of old channels is rampant everywhere. Copper wires are still an inexpensive option to transmit data in all directions. However, with the growing amount of data, transmission delivery requires a fast time so that the data does not experience delays. It occurs when streaming video using the internet. Videos that have an excellent quality will experience a dashed transmission.

The fastest data transmission at a previous time was to utilize electrical signals with copper wires. Then came the wireless technology with GSM signal which currently supports $4 \mathrm{G}$ technology. Fiber optic appears and is in high demand because its speed is much faster than the speed of electricity or sound. The transmission uses light at speeds of $300,000,000$ meters per second while the sound speed is only 343 meters per second.

Based on this speed, the fiber optic solution is used as the data carrier in the next era. However, in the construction of this network, many obstacles faced, especially in the field of costs incurred. This cable is very expensive, and if not calculated carefully, it will harm some parties including harming the state if the project is controlled by the state. This cabling requires a good algorithm to optimize cable requirements. The shortest route search is an action to reduce cost [5]. Prim's algorithm is one algorithm that can be used to calculate minimum cable usage to minimize cost. This algorithm will look for shorter connections between the two branching points of the cable. This use is expected to reduce the number of cables used on each transmitter pole.

\section{METHODS AND MATERIAL}

\subsection{Fiber Optic}

Fiber optic is a medium of information that is now used to connect computer networks. This transmission device is made of glass fiber and plastic that uses light bias in the process of sending information. The light source used is a laser. Laser becomes an option because it has a very narrow spectrum. The use of fiber optic cables is based on the speed at which data transmission. The emitted signal is not affected by electromagnetic waves as in copper wires. The induction will occur in copper cables when there are cables adjacent to other cables 
including the influence of radio frequency waves of communication. Fiber optic is used as the backbone of a network. It is an unstable wireless transmission if there is a radio frequency colliding [8].

Fiber optic has two modes, single and multi mode.

- Single-mode fibers have a small core that is 0.00035 -inch diameter or 9 microns and serves to transmit infrared laser light. The wavelength approximately 1300-1550 nanometers.

- Multi-mode fibers have a larger core that is 0.0025 inch diameter or 62.5 microns and serves to transmit infrared laser light with a wavelength of about 8501300 nanometers.

The single mode has a small core size, laser light source, unlimited bandwidth, and long distances. The multi mode has a larger core size, the source of the laser beam, the limited bandwidth, the distance of the beam is not so far away. The basic structure of fiber optic consists of three parts: core, cladding, and buffer/coating. Core and cladding are made of glass while the buffer is made of plastic to maintain flexibility.

Fusion Splicer is a tool for connecting glass-based optical fibers that implement an electric power that has been converted into a laser beam-shaped light media that serves to heat the broken glass on the core so that it reconnects properly. It must have high accuracy so that the cable at the time of splicing can be near perfect [9].

The process of sending information on fiber optic cable is by applying the welding of glass media, and there is a glass melting process that produces the connection without any gaps. This connection may fail if the cable connection does not follow the procedure properly. The connection process should be repeated in case of error. It aims to create damping below $0.25 \mathrm{~dB}$. Improper connection process will result in high Bit Error Rate. It results in high resistance and makes the transmission flow to the device will not run perfectly.

\subsection{Prim's Algorithm}

The minimum range tree is a way to form a link to the graph to all points with the smallest weights until the minimum distance is obtained [1][4][10].Prim's algorithm was discovered in 1930 by a mathematician named Vojtěch Jarník. Robert C. Prim and Dijkstra reexamined this algorithm separately in 1957 and 1959. Prim's algorithm is a method in graph theory to find the minimum spanning tree for an interconnected weighted graph [2][3]. It means that a subset of edges that form a tree containing nodes. It aims to minimize the overall weighting of all edges in the graph. If the graph is not connected, the graph has only one minimum range tree for one of the connected components [6][7].

Table 1. Initial weight

\begin{tabular}{|c|c|c|}
\hline Source & Dest. & Weight \\
\hline A & B & 4 \\
\hline & F & 5 \\
\hline B & A & 4 \\
\hline & C & 5 \\
\hline & E & 4 \\
\hline & F & 2 \\
\hline C & B & 5 \\
\hline & D & 6 \\
\hline & E & 2 \\
\hline & F & 3 \\
\hline D & C & 6 \\
\hline & E & 3 \\
\hline E & B & 4 \\
\hline & C & 2 \\
\hline & D & 3 \\
\hline & F & 10 \\
\hline F & A & 5 \\
\hline & B & 2 \\
\hline & C & 3 \\
\hline & E & 10 \\
\hline
\end{tabular}

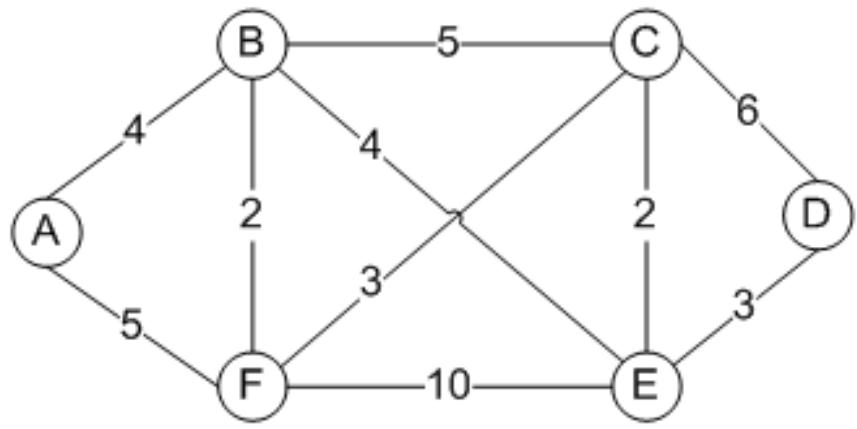

Figure 1. Initial weighted graph 
Table 1 and Figure 1 are preliminary data for weighted graphs. Six nodes are forming the graph, and some of the nodes are interconnected [11]. Be aware this node will get the shortest path to connect the nodes.

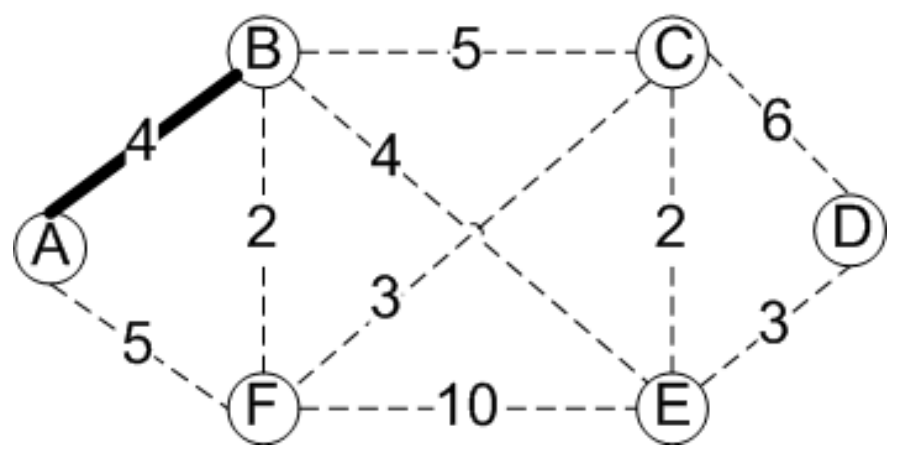

Figure 2. Step one

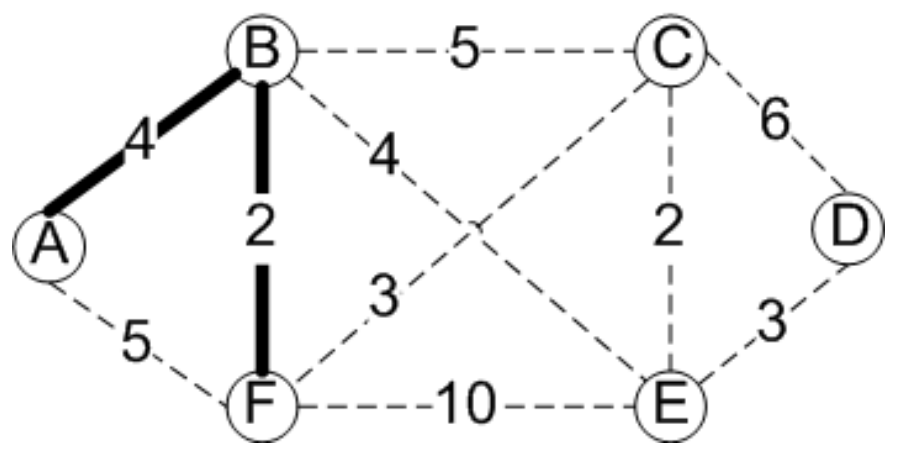

Figure 3. Step two

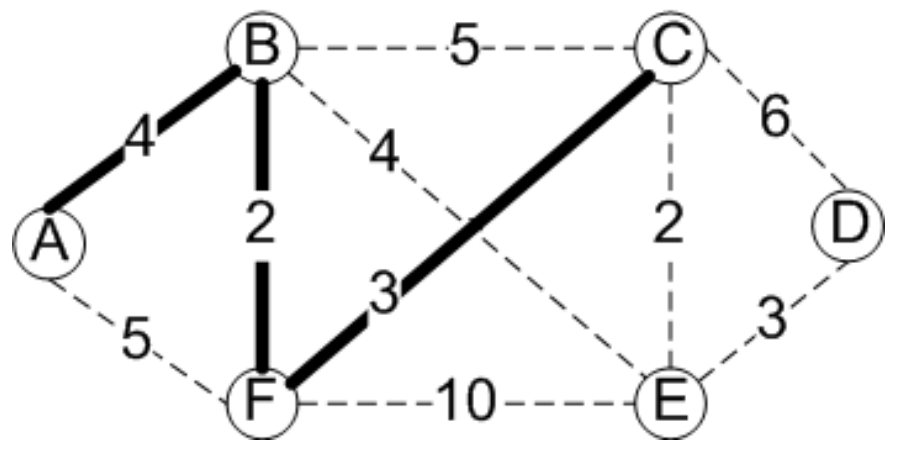

Figure 4. Step three

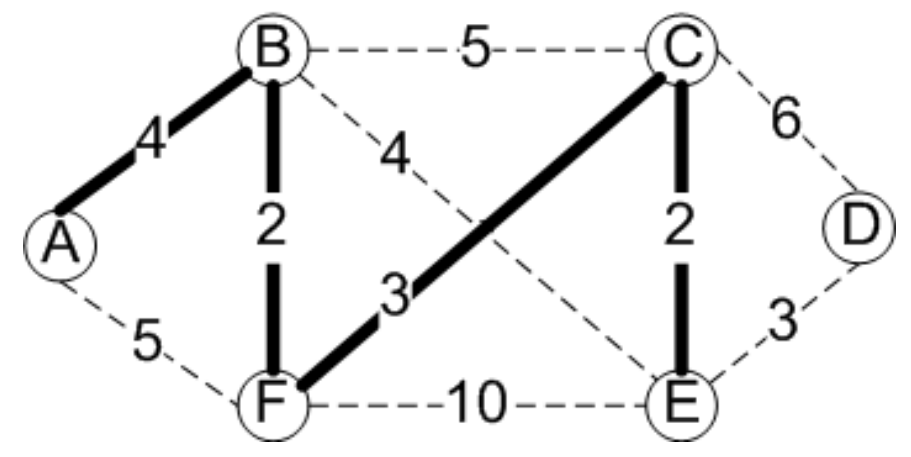

Figure 5. Step four

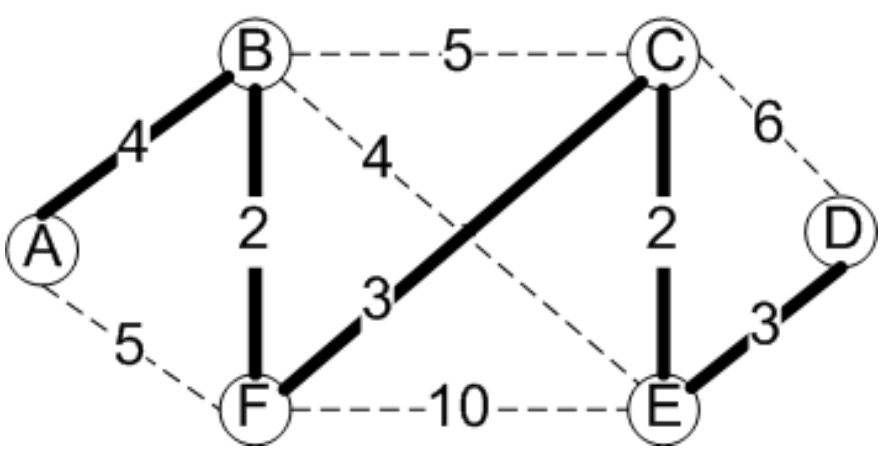

Figure 6. Step five

The node search starts from $\mathrm{A}$ to $\mathrm{F}$, so all nodes are connected. The use of Prim's algorithm works if there are nodes that have multiple branches. Otherwise, no algorithm needs to be applied. Figure 2 to 6 describes the process of determining which nodes will be the minimum spanning tree. The nodes that have the smallest weights will always be selected to be the next route. So the results obtained are A-B-F-C-E-D as in Figure 6.

\section{RESULTS AND DISCUSSION}

This test is trying to apply the optical fiber cable withdrawal in a particular area. There are 24 points to be linked directly. Some of the points are interconnected, so it should be determined which point further shortens the time and length. Table 2 describes the location of the coordinate points as examples of their application.

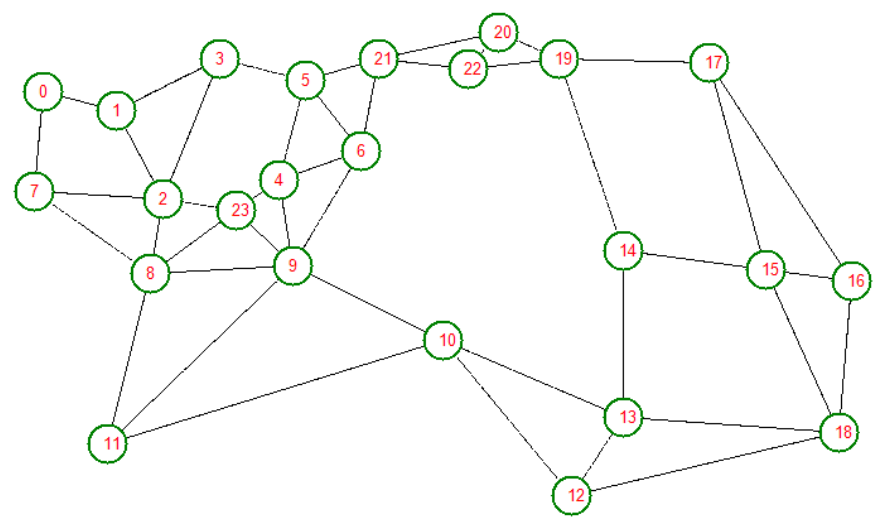

Figure 7. Fiber optic graph route 
Table 2. Fiber optic coordinate

\begin{tabular}{|c|c|c|l|}
\hline No. & $\mathbf{X}$ & $\mathbf{Y}$ & \multicolumn{1}{|c|}{ Branches } \\
\hline 0 & 22 & 29 & 1,7 \\
\hline 1 & 39 & 34 & $0,2,3$ \\
\hline 2 & 50 & 58 & $1,3,7,8,23$ \\
\hline 3 & 63 & 20 & $1,2,5$ \\
\hline 4 & 77 & 53 & $5,6,9,23$ \\
\hline 5 & 83 & 26 & $4,6,21$ \\
\hline 6 & 96 & 45 & $4,5,9,21$ \\
\hline 7 & 20 & 56 & $0,2,8$ \\
\hline 8 & 47 & 78 & $2,7,9,11,23$ \\
\hline 9 & 80 & 76 & $4,6,8,10,11,23$ \\
\hline 10 & 115 & 96 & $9,11,12,13$ \\
\hline 11 & 37 & 124 & $8,9,10$ \\
\hline 12 & 145 & 138 & $10,13,18$ \\
\hline 13 & 157 & 117 & $10,14,18$ \\
\hline 14 & 157 & 72 & $13,15,19$ \\
\hline 15 & 190 & 77 & $14,16,17,18$ \\
\hline 16 & 210 & 80 & $15,17,18$ \\
\hline 17 & 177 & 21 & $15,16,19$ \\
\hline 18 & 207 & 121 & $12,13,15,16$ \\
\hline 19 & 142 & 20 & $14,17,20,22$ \\
\hline 20 & 128 & 13 & $19,21,22$ \\
\hline 21 & 100 & 20 & $5,6,20,22$ \\
\hline 22 & 121 & 23 & 19,21 \\
\hline 23 & 67 & 61 & $2,4,8,9$ \\
\hline & & & \\
\hline
\end{tabular}

Figure 7 is a graph formed based on the coordinates shown in Table 8 . Each node will be connected directly to its branches. Each node intersection has weights that are calculated based on Euclidean Distance. For example, the weights from node 0 to 1 can be seen in the following calculations:

$$
\begin{aligned}
\mathrm{D}_{(0,1)} & =\sqrt{(x 1-x 0)^{2}+(y 1-y 0)^{2}} \\
& =\sqrt{(39-22)^{2}+(34-29)^{2}} \\
& =\sqrt{17^{2}+5^{2}} \\
& =\sqrt{289+25} \\
& =\sqrt{314} \\
& =17.7200451466693
\end{aligned}
$$

\begin{tabular}{|c|c|c|}
\hline Node 1 & Node 2 & Weight \\
\hline [0] & [1] & 17,72 \\
\hline [0] & [7] & 27,07 \\
\hline [1] & [0] & 17,72 \\
\hline [1] & [2] & 26,4 \\
\hline [1] & [3] & 27,78 \\
\hline [2] & [1] & 26,4 \\
\hline [2] & [3] & 40,16 \\
\hline [2] & [7] & 30,07 \\
\hline [2] & [8] & 20,22 \\
\hline [2] & [23] & 17,26 \\
\hline [3] & [1] & 27,78 \\
\hline [3] & [2] & 40,16 \\
\hline [3] & [5] & 20,88 \\
\hline [4] & [5] & 27.66 \\
\hline [4] & [6] & 20,62 \\
\hline [4] & [9] & 23,19 \\
\hline [4] & [23] & 12,81 \\
\hline [5] & [4] & 27,66 \\
\hline [5] & [6] & 23,02 \\
\hline [5] & [21] & 18,03 \\
\hline [6] & [4] & 20,62 \\
\hline [6] & [5] & 23,02 \\
\hline [6] & [9] & 34,89 \\
\hline [6] & [21] & 25,32 \\
\hline [7] & [0] & 27,07 \\
\hline [7] & [2] & 30,07 \\
\hline [7] & [8] & 34,83 \\
\hline [8] & [2] & 20,22 \\
\hline [8] & [7] & 34,83 \\
\hline [8] & [9] & 33,06 \\
\hline [8] & [11] & 47,07 \\
\hline [8] & [23] & 26,25 \\
\hline
\end{tabular}

Table 3. Weight result 


\begin{tabular}{|c|c|c|}
\hline [9] & [4] & 23,19 \\
\hline [9] & [6] & 34,89 \\
\hline [9] & [8] & 33,06 \\
\hline [9] & [10] & 40,31 \\
\hline [9] & [11] & 64,44 \\
\hline [9] & [23] & 19,85 \\
\hline [10] & [9] & 40,31 \\
\hline [10] & [11] & 82,87 \\
\hline [10] & [12] & 51,61 \\
\hline [10] & [13] & 46,96 \\
\hline [11] & [8] & 47,07 \\
\hline [11] & [9] & 64,44 \\
\hline [11] & [10] & 82,87 \\
\hline$[12]$ & [10] & 51,61 \\
\hline [12] & [13] & 24,19 \\
\hline [12] & [18] & 64,29 \\
\hline [13] & [10] & 46,96 \\
\hline [13] & [14] & 45 \\
\hline [13] & [18] & 50,16 \\
\hline$[14]$ & [13] & 45 \\
\hline$[14]$ & [15] & 33,38 \\
\hline [14] & [19] & 54,12 \\
\hline$[15]$ & [14] & 33,38 \\
\hline [15] & [16] & 20,22 \\
\hline [15] & [17] & 57,49 \\
\hline [15] & [18] & 47,17 \\
\hline$[16]$ & [15] & 20,22 \\
\hline [16] & [17] & 67,6 \\
\hline$[16]$ & [18] & 41,11 \\
\hline [17] & [15] & 57,49 \\
\hline [17] & [16] & 67,6 \\
\hline [17] & [19] & 35,01 \\
\hline$[18]$ & [12] & 64,29 \\
\hline$[18]$ & [13] & 50,16 \\
\hline [18] & [15] & 47,17 \\
\hline
\end{tabular}

\begin{tabular}{|l|l|l|}
{$[18]$} & {$[16]$} & 41,11 \\
\hline \multicolumn{3}{|c|}{} \\
\hline$[19]$ & {$[14]$} & 54,12 \\
\hline$[19]$ & {$[17]$} & 35,01 \\
\hline$[19]$ & {$[20]$} & 15,65 \\
\hline$[19]$ & {$[22]$} & 21,21 \\
\hline \multicolumn{3}{|c|}{} \\
\hline$[20]$ & {$[19]$} & 15,65 \\
\hline$[20]$ & {$[21]$} & 28,86 \\
\hline$[20]$ & {$[22]$} & 12,21 \\
\hline \multicolumn{3}{|c|}{} \\
\hline$[21]$ & {$[5]$} & 18,03 \\
\hline$[21]$ & {$[6]$} & 25,32 \\
\hline$[21]$ & {$[20]$} & 28,86 \\
\hline$[21]$ & {$[22]$} & 21,21 \\
\hline \multicolumn{3}{|c|}{} \\
\hline$[22]$ & {$[19]$} & 21,21 \\
\hline$[22]$ & {$[21]$} & 21,21 \\
\hline \multicolumn{3}{|c|}{} \\
\hline$[23]$ & {$[2]$} & 17,26 \\
\hline$[23]$ & {$[4]$} & 12,81 \\
\hline$[23]$ & {$[8]$} & 26,25 \\
\hline$[23]$ & {$[9]$} & 19,85 \\
\hline
\end{tabular}

Table 3 shows the weighting results based on the previously defined coordinates. After the weights are obtained, the Prim's algorithm process can be implemented. The minimum spanning tree results are as in Figure 8. If there are branches, then the weights are selected based on the smallest weights. The Greedy algorithm inspires it.

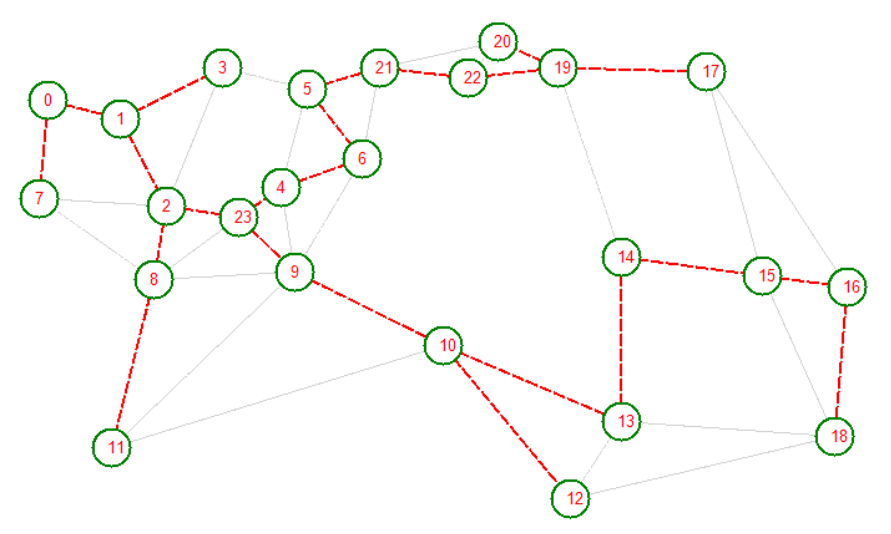

Figure 8. Prim's algorithm result 


\section{CONCLUSION}

Prim's algorithm performs searches on notes that have the shortest weights. This process can be done on fiber optic cabling. Due to very high cable prices, this algorithm has an important role to play in reducing construction costs. It always produces sides that are members of the minimum spanning tree. It makes every step of the search efficient and effective. However, in a graph that has many circuits, this algorithm takes a long time to check the existence of the circuit against the spanning tree that has been formed. In many branch graphs such as complete graphs, this method becomes an obstacle in the problem of graduation. This algorithm is considered good enough to be applied to the development of fiber optic lines so that the cost is not a waste.

\section{REFERENCES}

[1]. N. P. Akpan and I. A. Iwok, "A Minimum Spanning Tree Approach of Solving a Transportation Problem," International Journal of Mathematics and Statistics Invention, vol. 5, no. 3, pp. 09-18, 2017.

[2]. S. Dagar, "Modified Prim's Algorithm," International Journal of Computer and Information Technology, vol. 3, no. 2, pp. 26-29, 2012.

[3]. T. O. Arogundade, B. Sobowale and T. A. Akinwale, "Prim Algorithm Approach to Improving Local Access Network in Rural Areas," International Journal of Computer Theory and Engineering, vol. 3, no. 3, pp. 413-417, 2011.

[4]. A. Mandal, J. Dutta and S. Pal, "A New Efficient Technique to Construct a Minimum Spanning Tree," International Journal of Advanced Research in Computer Science and Software Engineering, vol. 2, no. 10, pp. 93-97, 2012.

[5]. A. P. U. Siahaan, "Heuristic Function Influence to the Global Optimum Value in Shortest Path Problem," IOSR Journal of Computer Engineering, vol. 18, no. 5, pp. 39-48, 2016.

[6]. M. Jose and S. Sreekumar, "Comparison of BFS and Prim's Algorithm when Used in MANETs Routing," International Journal of Computer Applications Technology and Research, vol. 5, no. 2, pp. 92-94, 2016.
[7]. W.-C. Chang, T.-H. Wang and Y.-D. Chiu, "Board Game Supporting Learning Prim's Algorithm and Dijkstra's Algorithm," International Journal of Multimedia Data Engineering and Management, vol. 1, no. 4, pp. 16-30, 2010.

[8]. A. Amaku, R. E. Watti and J. Joshua, "Optic Fiber as a Reliable Medium for Metropolitan Area Networking (MAN) Connectivity," International Journal of Engineering and Technology, vol. 4, no. 9, pp. 542-547, 2014.

[9]. P. Sharma, R. K. Arora, S. Pardeshi and M. Singh, "Fibre Optic Communications: An Overview," International Journal of Emerging Technology and Advanced Engineering, vol. 3, no. 5, pp. 474-479, 2013.

[10]. A. Kumar and G. Kumar, "An Efficient Method to Construct Minimum Spanning Tree," International Journal of Latest Technology in Engineering, Management \& Applied Science, vol. 4, no. 10, pp. 48-51, 2015.

[11]. Ray, "Algoritma Prim," 20 April 2011. [Online]. Available:

http://architectureandrouting.blogspot.co.id/ 2011/04/algoritma-prim.html. [Accessed August 2017]. 